\title{
Poïesis et technologie de l'enchantement dans la « maison-stock » d'Olivier Jullian à Nîmes
}

\section{Clara Breteau}

\section{(2) OpenEdition}

\section{Journals}

Édition électronique

URL : https://journals.openedition.org/cdg/5692

DOI : $10.4000 /$ cdg.5692

ISSN : 2107-7266

Éditeur

UMR 245 - CESSMA

\section{Référence électronique}

Clara Breteau, «Poïesis et technologie de l'enchantement dans la « maison-stock » d'Olivier Jullian à Nîmes », Carnets de géographes [En ligne], 14 | 2020, mis en ligne le 01 décembre 2020, consulté le 22 mai 2021. URL : http://journals.openedition.org/cdg/5692 ; DOI : https://doi.org/10.4000/cdg.5692

Ce document a été généré automatiquement le 22 mai 2021.

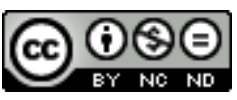

La revue Carnets de géographes est mise à disposition selon les termes de la Licence Creative Commons Attribution - Pas d'Utilisation Commerciale - Pas de Modification 4.0 International. 


\section{Poïesis et technologie de} l'enchantement dans la « maisonstock » d'Olivier Jullian à Nîmes

\section{Clara Breteau}

Figure 1 : Maison d'Olivier Jullian, vue de la façade

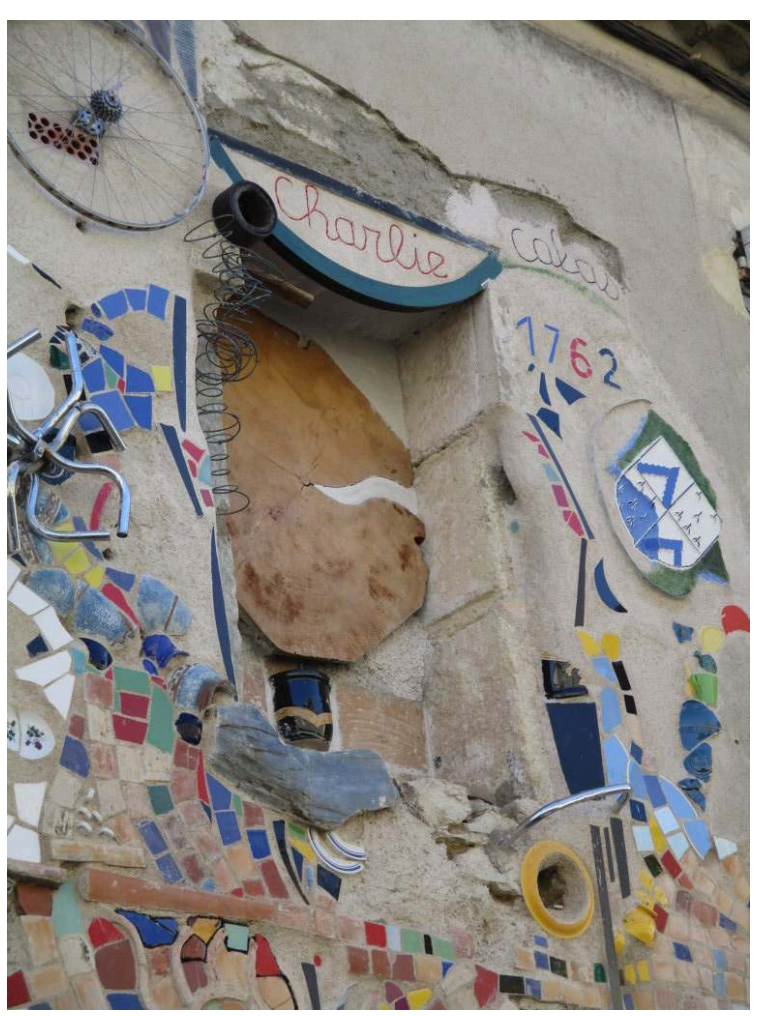

Source/crédit : Breteau, 2015 


\section{Introduction}

1 Dans Écritures révolutionnaires de la nature au xix siècle, Bertrand Guest montre comment des pionniers de la géographie tels qu'Alexander Von Humboldt ou Élisée Reclus rendent manifeste le lien inaugural et profond de la discipline avec la poésie. «[Refusant] le hiatus croissant entre la science tendant à s'autonomiser et la pensée poétique du cosmos", auteurs de "livres aussi scientifiques que poétiques [...] [reliant] [...] la géographie de la terre à celle de l'Homme », ils apparaissent «à la fois [comme] les poètes et les scientifiques de [la] description moderne du monde $»^{1}$ (Guest, $2003: 12$, 14, 27 et 287). À travers le $\mathrm{xx}^{\mathrm{e}}$ siècle, alors que la géographie développe des outils quantitatifs pour se voir reconnaître comme "science dure", émerge un autre mouvement appelant à une géographie «humaniste» qui continue d'attribuer à l'entreprise géographique une dimension poétique irréductible. L'un de ses précurseurs, Éric Dardel, considère ainsi dans L'Homme et la Terre que - parce que le ou la géographe s'engage dans la description de la «relation concrète [...] [qui] se noue entre l'Homme et la Terre » - «[son] langage devient sans effort celui du poète » (1952:2-3).

2 Alors que des courants mobilisant des notions telles que le mode d'habiter (MorelBrochet et Ortar, 2012) et l'habitabilité (Blanc, 2010) voient toujours une forme d'«activité poétique» dans la création de milieux de vie «habitables» et l'appropriation des lieux (Blanc, Lamarche, 2015 : 14), d'aucuns soulignent l'importance de remettre au cœur de la fabrique de la ville «les liens poétiques des citadins à leur territoire " (Bailly, 2016) et tous ces "processus créatifs» et "singuliers» qui composent «la poétique» de nombre d'espaces ou d'objets géographiques (Le Gall, Rougé, 2014:1) . On observe par ailleurs l'ouverture récente à la géographie et à l'urbanisme d'une " écopoétique " (Meillon et al., 2018) longtemps cantonnée à l'étude quasi exclusive des liens entre corpus littéraires et environnement (Blanc et al., 2017). Rassemblées sous l'ombrelle des "humanités environnementales" autour d'objets transdisciplinaires tels que "le sauvage urbain» (Meillon et al., 2018), littérature, géographie, urbanisme, architecture et anthropologie entament des dialogues féconds, que ce soit au sein de colloques "écopoétiques " ${ }^{2}$ ou à l'intérieur même de collectifs de recherche interdisciplinaires parfois associés au courant de la géopoétique (White, 2009 ; Bouvet, 2015). S'appliquant, dans le cadre théorique des nouveaux matérialismes (Dolphijn, Van der Tuin, 2012), à l'étude des "enchevêtrements " entre matière et signification observables sur le terrain d'habitats autonomes alternatifs, cet article et les recherches dont ils procèdent (Breteau, 2018) ${ }^{4}$ 'inscrivent dans une telle tentative écopoétique de faire dialoguer géographie humaine, concepts philosophiques, épistémologie des sciences sociales et notions de théorie littéraire autour d'un même objet : les habitats alternatifs autonomes ${ }^{5}$.

3 Expérimentant des formes de vie et de survie en-dehors de la société industrielle productiviste, ces habitats axés sur l'autonomie matérielle s'affranchissent de l'industrie agro-alimentaire, du marché du logement et des énergies fossiles pour réinscrire leurs moyens de survie dans des réseaux d'entraide locaux et dans le milieu naturel qui les environne (Pruvost, 2013). Cependant, pour être compris, ces lieux nécessitent d'aller au-delà de la description technique du « kit de l'autonomie » qui les accompagne. Ils demandent aussi de creuser plus loin que les profils socio-économiques et parcours de vie individuels de leurs habitants. En effet, ces habitats autonomes 
développent également des «cultures de nature » (Blanc, 1995 : 14) qui racontent des histoires et débouchent sur des façons singulières de faire signe et faire sens du monde, faisant se fondre ensemble langage et habitat.

4 Étudiées sur le terrain pendant un séjour itinérant de six mois à travers 24 lieux ${ }^{6}$, ces cultures de nature apparaissent marquées par une prolifération végétale et animale, une multiplication des tas, stocks et composts et tout un entrelacement d'usages et d'activités qui accouche de machines, d'espaces et de pratiques étranges et poétiques. Véritable "métier à tisser", l'habitat autonome redonne alors un visage concret au leitmotiv de "l'habitation poétique du monde» abordé jusqu'à présent dans la littérature de manière essentiellement théorique (Heidegger, 1958 : 224-245; Berque et al., 2012 ; Collot, 2014 : 109 ; Lefebvre, $1970: 133$, 155). En effet, on y voit aussi émerger un type de faire singulier que nous avons baptisé « poïesis». Une étude lexicologique et philosophique des mobilisations de cette notion dans l'histoire des idées montre notamment que la poïesis a depuis longtemps été utilisée pour désigner, non seulement la création littéraire ou la création tout court, mais aussi un mode de production naturel, organique et spontané (Liddel et al., 1996 : 1427-1429). Traversés par ce mode de production, les retissages et rebouclages que l'on observe chez les habitants n'apparaissent plus juste comme « métaboliques " $^{7}$. Ils se transposent et se propagent à une multitude d'autres niveaux, sur la trame esthétique des lieux tout d'abord, où l'on voit se multiplier les motifs textiles. Mais ils touchent aussi à quelque chose de plus profond, puisqu'ils déterminent également le surgissement dans l'habitat d'images singulières. Enchevêtrant intérieur et extérieur, humain et non-humain, sens et sensible, ces images font figure de points-clefs à travers lesquels la relation de l'être humain au monde se modifie.

5 Alors que la plupart des lieux de vie étudiés sont des habitats ruraux ou périurbains ${ }^{8}$ entourés d'un milieu naturel où les habitants puisent de nombreuses ressources, nous proposons d'étudier dans cet article le seul cas d'habitat hyperurbain figurant dans notre échantillon, en l'interrogeant plus précisément comme manifestation d'un certain type de "sauvage urbain » (Meillon et al., 2018). Cet habitat autorénové qui voit la quête d'autonomie se concentrer sur le plan de la construction fonctionne toutefois de manière couplée avec une autre maison autoconstruite par Olivier Jullian en milieu rural et dotée d'un profil d'autonomie plus complet s'exerçant également sur les plans alimentaire et énergétique. Malgré son degré «partiel» d'autonomie matérielle, la maison se rattache néanmoins aux autres habitats autonomes de notre échantillon du point de vue du langage singulier qui émerge dans le sillage de l'auto-rénovation.

6 Nous verrons en effet qu'une série d'enchevêtrements " métaboliques " ${ }^{9}$ se mettent en place, à partir desquels se développent des entrelacements esthétiques complexes et inépuisables formant ce que l'on peut appeler avec l'anthropologue Alfred Gell une "technologie de l'enchantement»(Gell, 2009: 92). Nous rentrerons alors dans la fabrique de ces différents tissages en nous penchant sur le type de faire spécifique ou poïesis qui se déploie à travers le lieu. Nous essaierons à travers cela, en écho à la tendance croissante qui voit des universitaires travailler des problématiques écologiques à la croisée des sciences sociales et littéraires (Meillon et al., 2018), de montrer que le courant écocritique longtemps cantonné au domaine littéraire (Blanc et al., 2017) peut être étendu à la géographie culturelle et à des formes de créativité « en actes ". 


\section{Des enchevêtrements métaboliques}

7 Une des premières caractéristiques des lieux de vie autonomes que nous avons étudiés est qu'ils retissent ensemble les flux d'énergie et de matière à l'intérieur de l'habitat, opérant un certain nombre d'entrelacements que nous qualifierons ici de «métaboliques ». Ceux-ci se traduisent à la fois par la recherche d'une prolifération d'espèces animales et végétales, par la conception bioclimatique des habitats ${ }^{10}$ et par des tissages " fonctionnels ", à savoir que les différents usages des espaces ou appareils sont hybridés et conçus en synergie les uns avec les autres. Enfin, ces enchevêtrements se manifestent par une concentration sur place des ressources, matériaux et matières premières et donc par une multiplication des stocks. Ceux-ci se retrouvent partout à travers le terrain, parfois dans les murs mêmes des habitats pour garantir leur isolation, et parfois à l'échelle de maisons entières, comme en témoigne le cas que nous allons étudier. Chez Olivier Jullian, à Nîmes, cette dernière caractéristique se voit en effet particulièrement exacerbée. À force d'intégrer des objets et rebuts aux murs de sa maison, Olivier Jullian en a fait ainsi une véritable « maison-stock».

Figure 2 (A, B, C et D) : Le tour du visiteur, vues sur les quatre façades de la maison d'Olivier Jullian

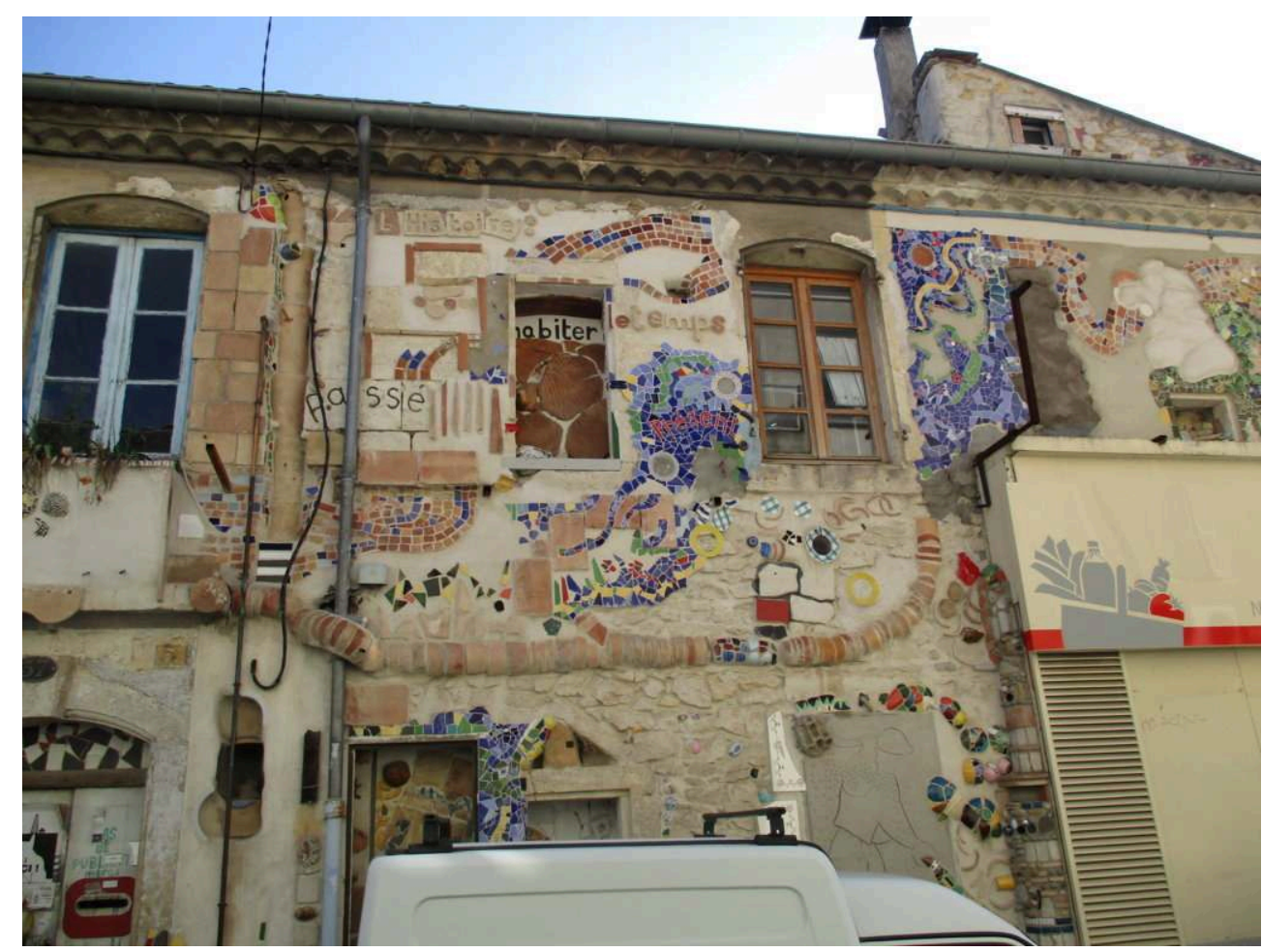



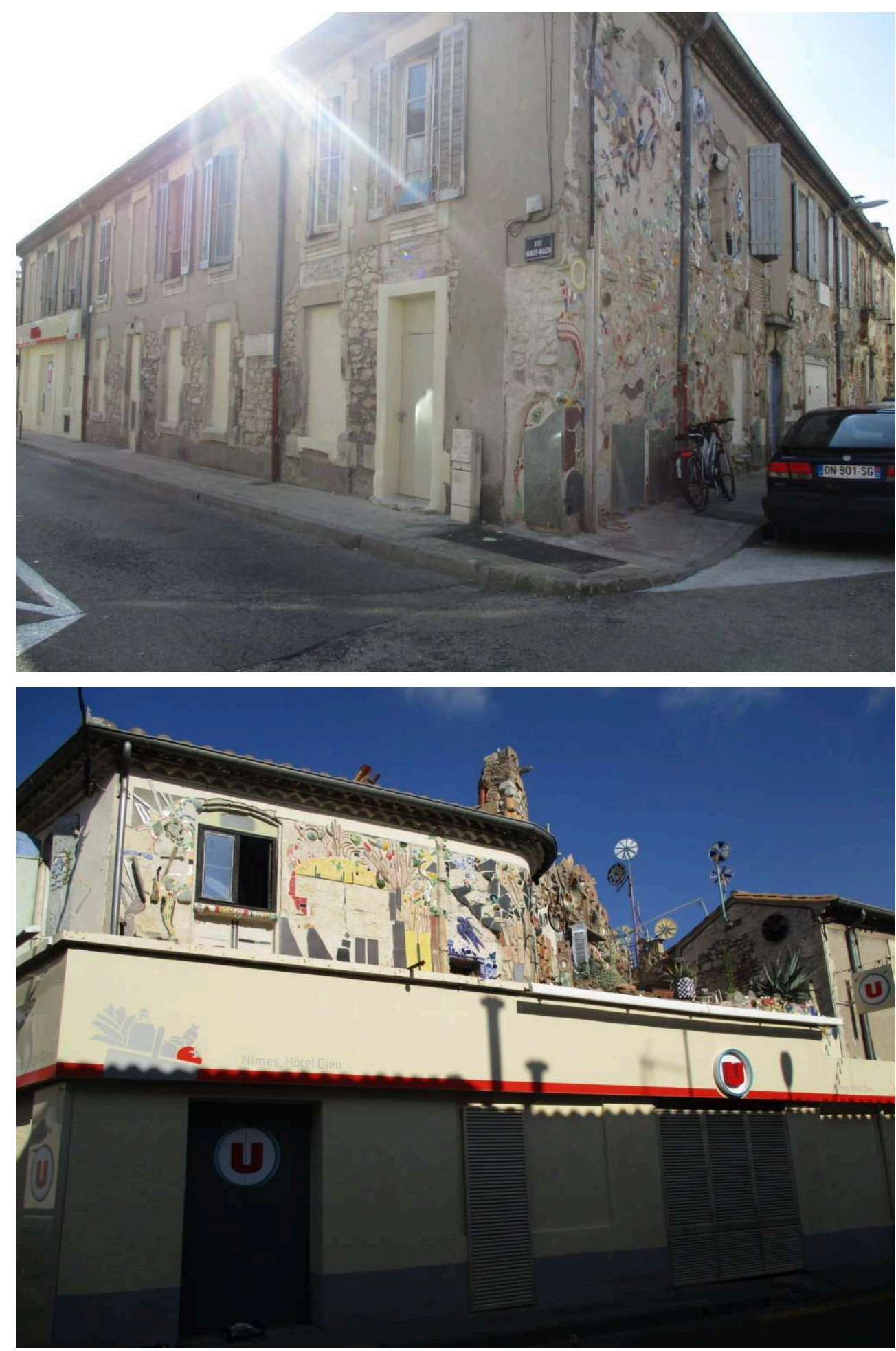


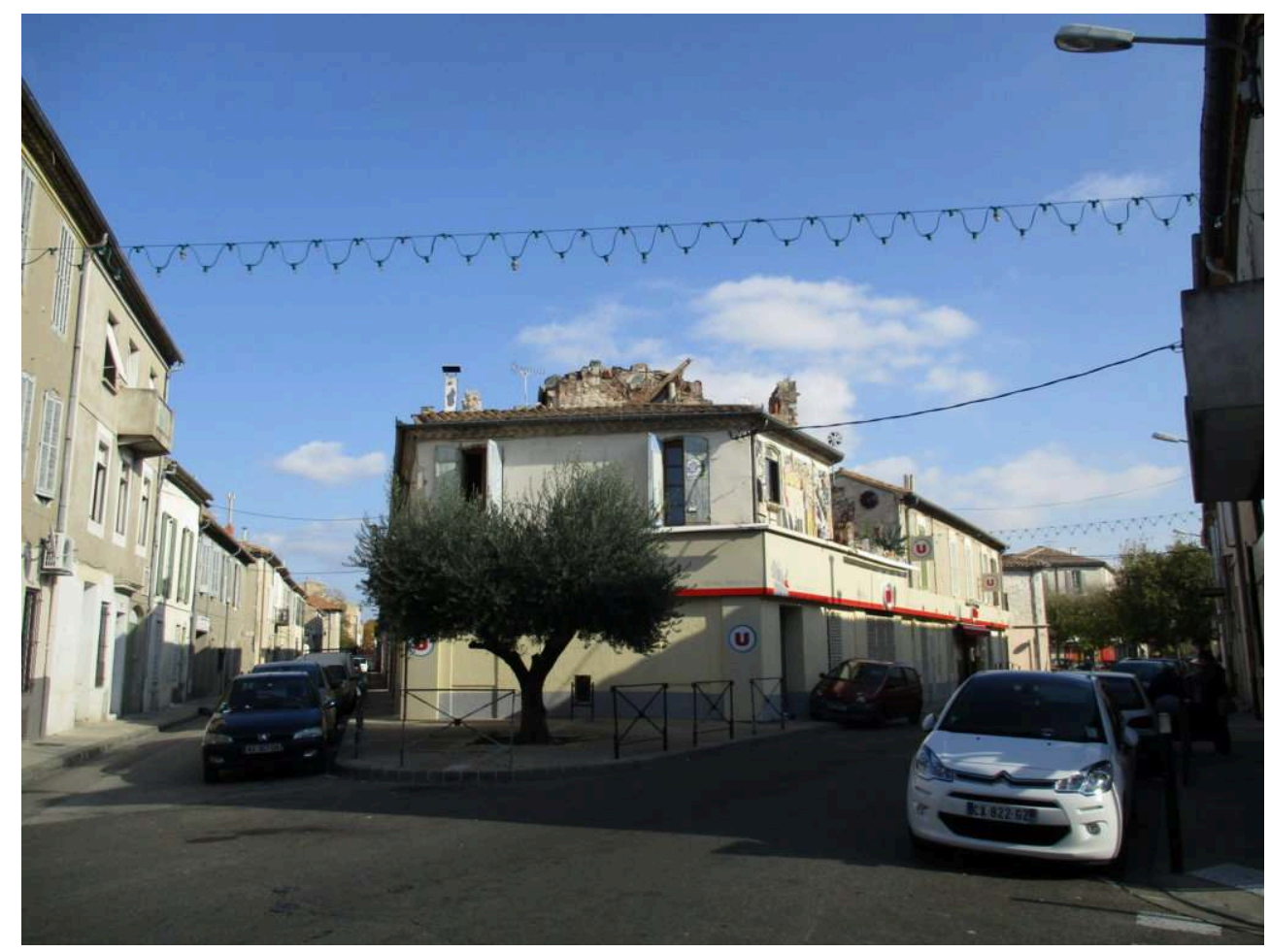

Source/crédit : Breteau, 2015

8 Une première observation permet tout d'abord de constater que la dimension de «stock » est inscrite dans l'architecture même de la maison. Celle-ci recouvre en effet tout un îlot urbain et se présente ainsi d'emblée comme un agrégat ou "pâté de maisons ». Se déployant sur quatre façades, elle contient sept appartements et un local commercial loué à l'enseigne de supermarché U. Olivier Jullian est propriétaire de la moitié du bâtiment, l'autre moitié appartenant à ses sœurs. Des désaccords avec ces dernières ont entraîné le blocage partiel des travaux de réfection des espaces intérieurs et le report de l'attention et des efforts d'aménagement d'olivier Jullian sur l'extérieur du bâtiment (figure 1). C'est à travers cet investissement très particulier des façades que nous allons pouvoir observer les rebouclages métaboliques qui, au-delà de l'architecture générale, donnent au bâtiment son statut de " maison-stock ».

Dans le détail, hormis de rares éléments langagiers ou picturaux (inscriptions, dates, dessins), la façade se révèle en effet incrustée d'une myriade d'objets et de fragments correspondant à différents types et échelles de rebouclages matériels (figure 3). On y trouve à la fois des éléments bâtimentaires (tuiles, tommettes, briques, mosaïque), des éléments techniques (guidons, roues, pneus, pédales de vélo, fil de fer, barreau d'échelle), des éléments domestiques (tasses, assiettes, théières, ressorts de matelas) et enfin des éléments naturels (plantes, sections de troncs, pierres). 
Figure 3 : Différents types d'enchevêtrements métaboliques
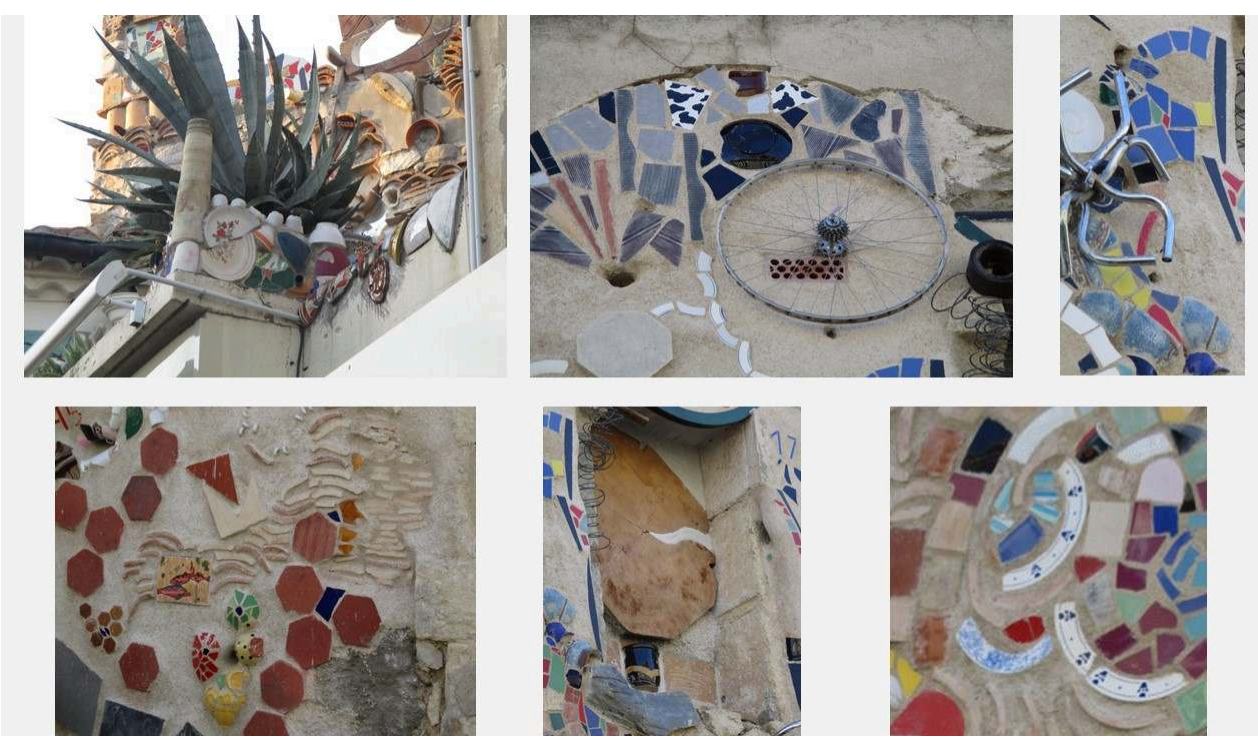

Source/crédit : Breteau, 2015

Comme le raconte Olivier Jullian, tout a commencé avec le chantier de réfection du toit et sa volonté de faire quelque chose des vieilles tuiles qu'il enlevait et entassait sans savoir qu'en faire : « on les a jetées les trois premiers jours et après j'ai dit on arrête, on les stocke. Et pour diminuer le stock, j'ai fait ça $»^{11}$. Ainsi, au lieu de se débarrasser des tuiles et d'en faire des "externalités » négatives bonnes pour la décharge, olivier Jullian les réintègre et les réassimile dans le corps du bâtiment. Il commence d'abord par les assembler en un petit fronton sur la façade ouest (figure 4), puis ses compositions s'étendent en surface : «t'imagines pas le nombre de tuiles qu'il y a. [...] Je voulais utiliser ces tuiles, et je voulais faire un truc original $»^{12}$. 
Figure 4 : Le petit fronton au poisson, première facette de la maison-stock

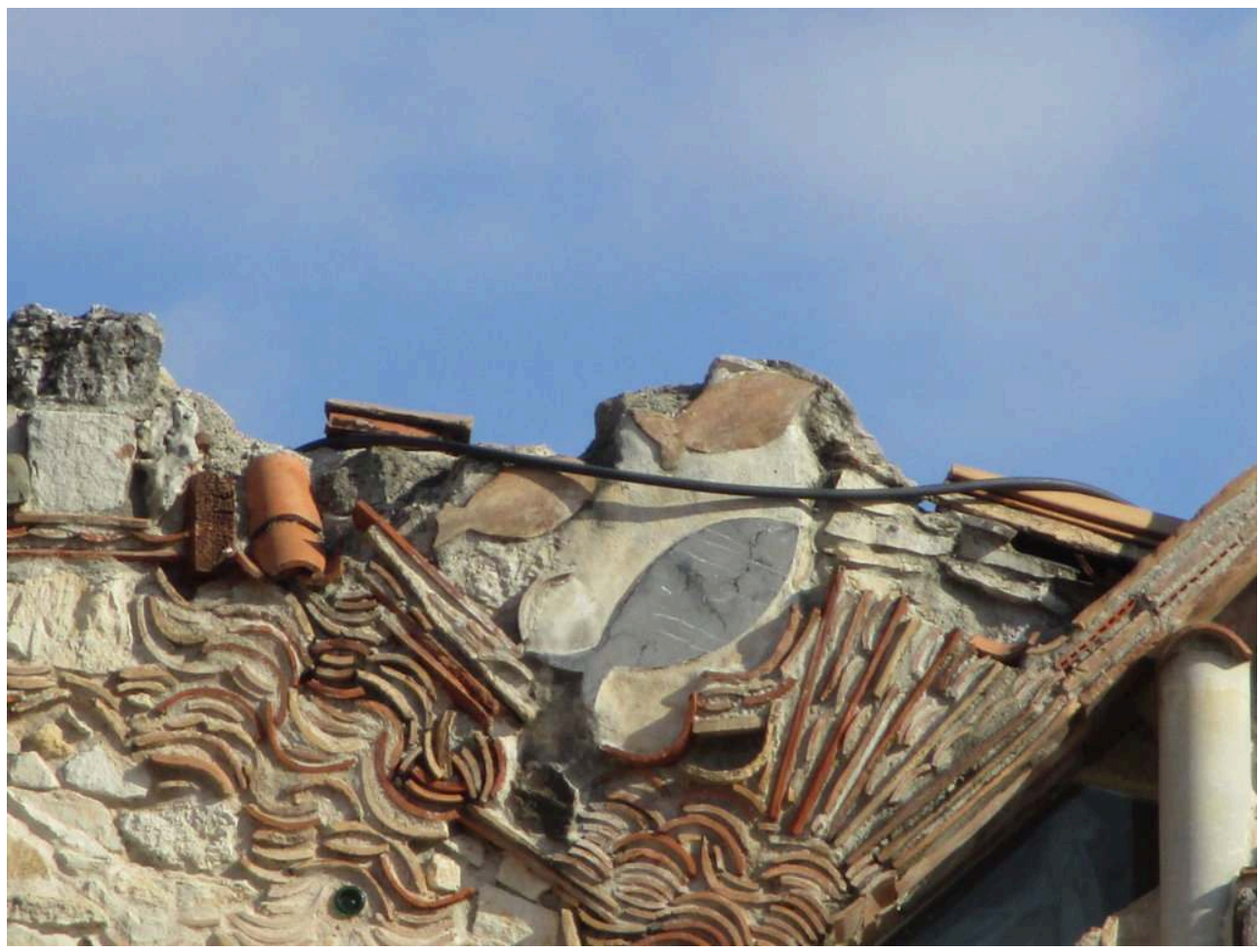

Source/crédit : Breteau, 2015

11 Ce faisant, la démarche d'Olivier Jullian se dote d'une portée politique. En effet, ce dernier déclare vouloir progressivement, par ce jeu de récupération, recouvrir l'intégralité du bâtiment, y compris la façade des locaux loués à l'enseigne de supermarché U. Il se trouve par-là engagé dans une bataille qui s'exerce non seulement contre l'enseigne mais aussi contre l'économie productiviste et la conception industrielle, rationaliste et court-termiste de l'«utile» qu'elle représente (le supermarché tirant en effet son nom « U » de ce même mot). Il est d'ailleurs intéressant de constater que le recyclage strictement matériel se voit redoublé sur le plan des signifiants par le fait que les deux mots "tuile» et "utile" sont de parfaites anagrammes. Par son refus de jeter et par l'impératif d'utilisation qui s'érige chez lui en principe de construction, Olivier Jullian vient donner à la notion d'usage une extension temporelle et spatiale niée par une société de consommation ayant donné la primauté à la valeur d'échange, à la croissance économique et à l'obsolescence programmée.

12 Alors que les opérations d'olivier Jullian s'élargissent progressivement à une multitude d'objets, la maison se reboucle au fur et à mesure sur elle-même, se feuillette ${ }^{13}$. Les objets qui s'y agrègent se multiplient et se diversifient, insérant le bâtiment dans des flux de matières à différentes échelles. Olivier Jullian va en effet intégrer aux murs d'autres éléments de la maison - beaucoup de pièces de son atelier vélo et bricolage notamment. Mais il va aussi utiliser des objets et toutes sortes de vaisselle cassée multicolore que les habitants du quartier, enthousiasmés par les fresques qu'ils voient naître sous leurs yeux, viennent lui apporter spontanément. La maison se retrouve ainsi enchevêtrée au corps de son quartier, qu'elle agrège par petits fragments. Cependant, elle va aussi assimiler et digérer des éléments d'un corps encore plus grand. En effet, Olivier Jullian utilise également dans ses compositions des matériaux 
provenant de son autre maison, une maison rurale, autonome et autoconstruite, d'où il ramène des troncs, des branches, des pierres ou encore du sable. Ainsi, le corps de l'habitat se renforce et s'épaissit de ses différentes enveloppes et milieux - du milieu socio-technique du quartier au milieu naturel du territoire rural dans lequel la ville s'inscrit et où se situe la seconde construction d'Olivier Jullian.

\section{Des enchevêtrements esthétiques}

Cependant, les rebouclages effectués à l'échelle de la maison ne sont pas juste matériels. En effet, celui-ci ne " cache " pas ou «n'invisibilise " pas les éléments qu'il recycle - et s'il y a stock, celui-ci se voit aussi doté d'une fertilité "esthétique». Comme l'a suggéré Lévi-Strauss dans La Pensée sauvage à propos des fonds de bricolage, la maison se retrouve "le résultat contingent de toutes les occasions qui se sont présentées de renouveler, d'enrichir le stock ou de l'entretenir avec les résidus de construction et de destruction antérieurs » $(1962: 30-31)$. En tant que tel, le bâtiment se libère alors des échanges strictement métaboliques qui l'ont produit et se fait l'abri ou le « nid» de nouvelles formes au potentiel d'association renouvelé ${ }^{14}$ (figure 5).

Figure 5 : Un « nid » de formes et associations renouvelées

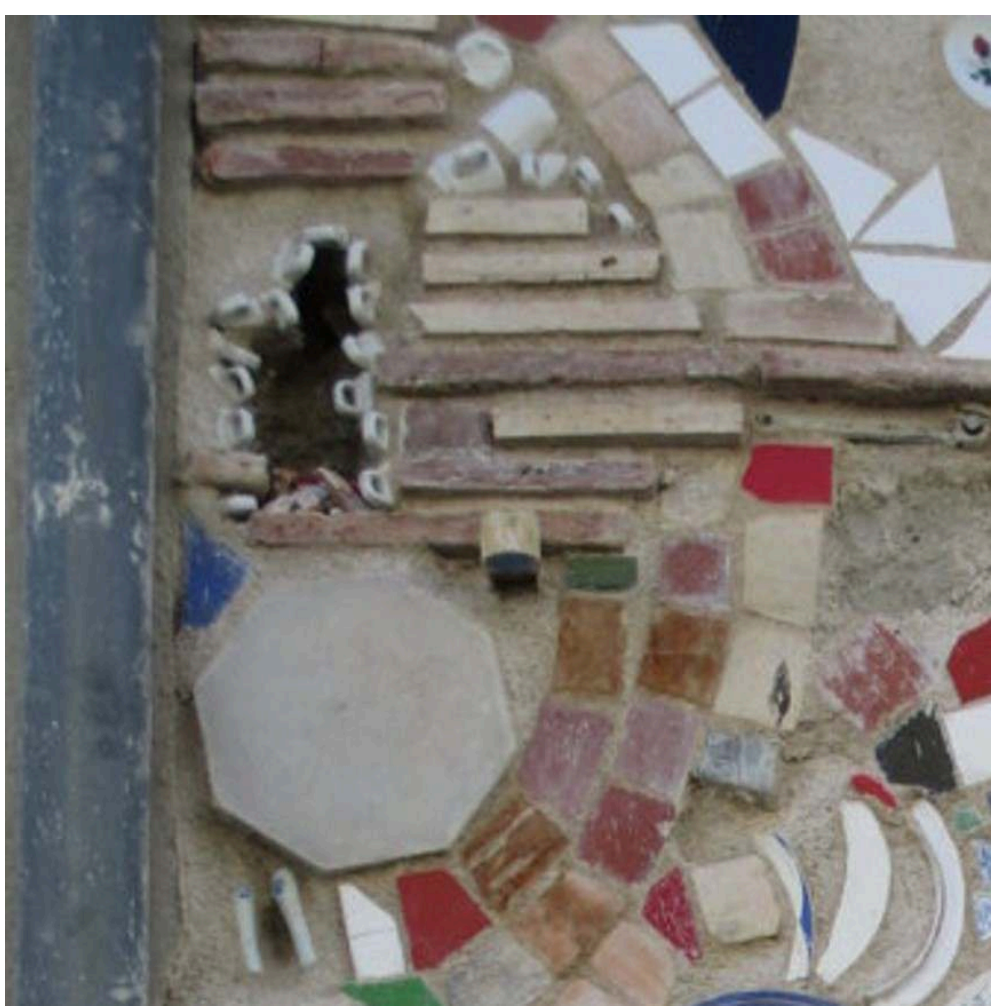

Source/crédit : Breteau, 2015

Ainsi, les retissages strictement physiques se transposent à d'autres niveaux - sur la trame esthétique des lieux tout d'abord. On voit notamment se multiplier dans le corps de l'habitat les motifs textiles, à commencer par la grande "toile de Pénélope » qui " prend en écharpe » le bâtiment (figure 6) et qu'Olivier Jullian a réalisée en référence aux conférences de Jean-Pierre Vernant sur l'Odyssée : 
J'ai fait partir la toile qu'elle tisse le jour et détruit la nuit avec ces petits carrés de tuiles, ce sont des bouts de tuiles coupées, avec un peu de bleu, de jaune... ça passe derrière, ça ressort, ça re-rentre dans la Méditerranée, ça ressort ici, [...] et ça va prendre le bâtiment en écharpe. ${ }^{15}$

Figure 6 : La toile de Pénélope

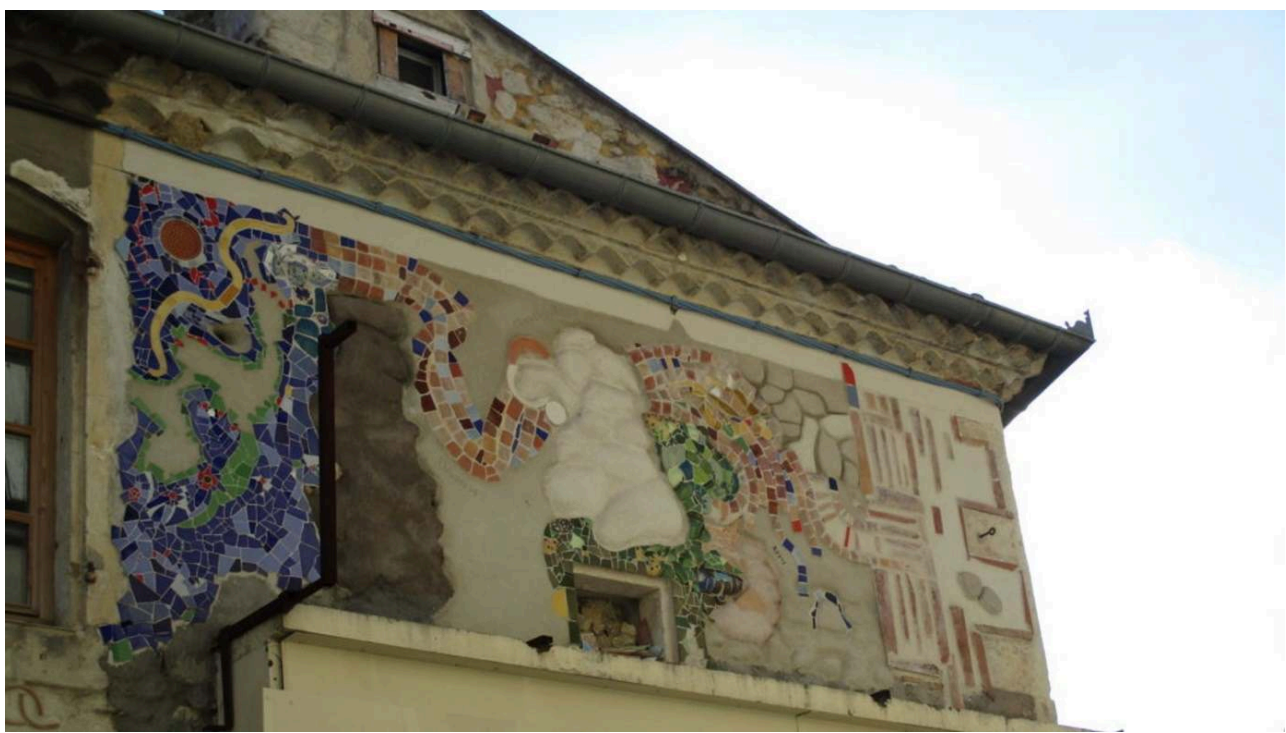

Source/crédit : Breteau, 2015

Le tissu d'échanges qui a présidé à l'apparition des fresques devient donc imagé, matérialisé et "filé » à travers l'habitat (figure 7). Il se manifeste aussi à travers l'intégration aux murs de différentes cordes, entrelacs et arabesques. On observe également une multiplication des roues et motifs cycliques qui font bien sûr écho au rouet de Pénélope mais viennent aussi métaphoriser les opérations de recyclages dont les fresques sont issues (figure 7).

Figure 7 : Une déclinaison de motifs textiles et cycliques
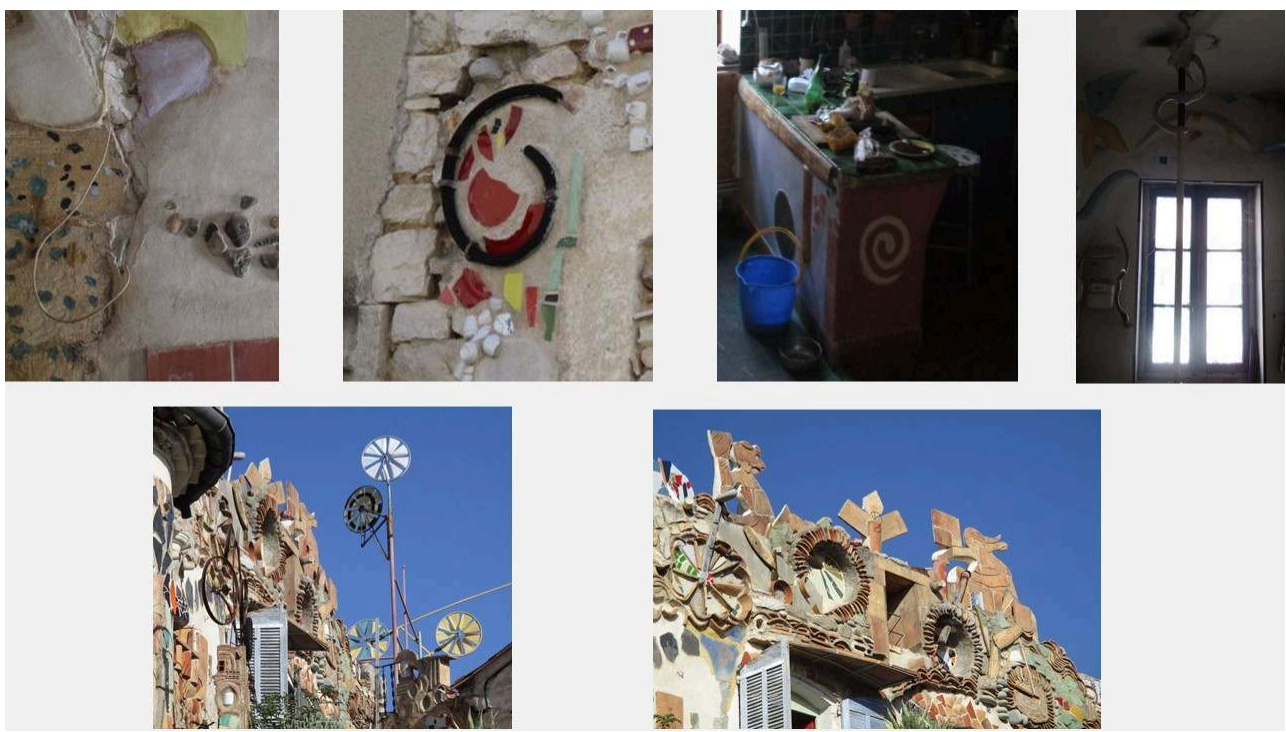

Source/crédit : Breteau, 2015 
16

$$
\begin{aligned}
& \text { traduit par le déploiement de motifs multifacettes qui, de par leur dimension } \\
& \text { proliférante et «buissonnante ", se rapprochent des motifs complexes aux qualités } \\
& \text { "adhésives » décrits par l'anthropologue Alfred Gell (2009). Par leur intermédiaire, la } \\
& \text { maison d'Olivier Jullian « enchaîne » ses visiteurs qui se retrouvent «pris dans [leurs] } \\
& \text { crochets et dans [leurs] épines » (Ibid.: 102). }
\end{aligned}
$$

Enfin, la transposition des enchevêtrements métaboliques au niveau esthétique se

Figure 8 : Des motifs multifacettes qui « enchaînent » les visiteurs

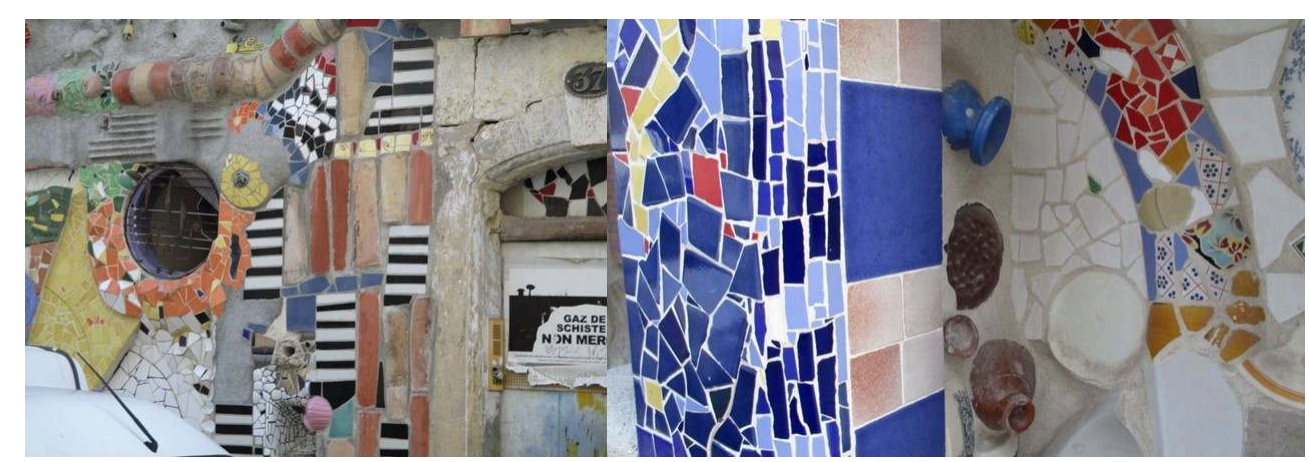

Source/crédit : Breteau, 2015

On observe alors un lieu qui s'ensauvage dans les formes de son «bricolage » (LéviStrauss, $1962: 30-31$; Loubes, 2010), mais aussi dans son esthétique et dans la relation qu'il tisse avec son visiteur. Par le jeu de ses motifs enchevêtrés et multifacettes, on peut estimer que la maison devient elle aussi porteuse de cette "technologie de l'enchantement» (Ibid.:92,103) que Gell définit comme la capacité qu'ont certains motifs complexes à « nouer des relations durables avec les personnes, car pour l'esprit humain, [ils renvoient] toujours à une opération cognitive inachevée. L'œil voit toujours d'abord une relation particulière, puis une autre, et cela à l'infini. [Leur] richesse est inépuisable. Les motifs ralentissent l'acte de la perception, l'arrêtent même, si bien qu'on ne les possède jamais complètement, on ne cesse de se les approprier » (2009: 100).

Quelque chose de sauvage dans le sens d'«indomptable» - qu'on ne peut jamais s'approprier totalement - surgit donc dans la trame esthétique du bâtiment. Ceci se reflète bien d'ailleurs dans la relation qu'entretiennent avec elle des habitants du quartier qui, selon Olivier Jullian, aiment la maison et sont fascinés par elle mais cependant n'arrivent pas à la « lire $»^{16}$.

\section{Un type de faire « poïétique » ouvert au hasard et au vivant}

Un tel phénomène se voit en partie expliqué et renforcé par le type de faire organique, ouvert au hasard et au vivant qui s'exerce à travers la maison. Présent à travers l'ensemble des habitats autonomes que nous avons étudiés, celui-ci n'apparaît réductible à aucune des grandes catégories traditionnelles du faire que sont l'artisanat, le bricolage, l'art ou encore l'ingénierie. Par-delà ce trouble et cette informité qui marquent le faire autonome, certains rapprochements se dessinent pourtant avec la poïesis, une notion bien plus complexe que celle de création «ex-nihilo » à laquelle elle 
se voit souvent rapportée (Michel, $2010: 256$ ) et dont nous avons reconstitué les trajets dans l'histoire des idées (Breteau, 2018 : 55-68).

La poïesis est liée dans ses racines indo-européennes à la construction de l'habitat et à l'aménagement ${ }^{17}$, et se trouve rattachée en Grèce ancienne à une notion de production définie de manière particulièrement large allant de la manufacture et des arts appliqués à la forge, la construction (Liddel et al., 1996 : 1427) et l'artisanat (Dicks, 2011 : 58 ; Ingold, Hallam, 2007). Elle englobe ainsi tout « un monde de labeur et [un] type de travaux particulièrement prosaïques » (Jauss, Shaw, 1982: 591) s'exerçant à l'échelle très locale de l'oikos dans le cadre d'une économie domestique. Cependant, la notion s'avère d'une plasticité telle qu'elle se voit appliquée plus tard aux chants et aux travaux littéraires (Platon, $2007: 146-147$ ).

21 Néanmoins, c'est à travers un autre faisceau de sens qui la voit se rattacher à des visages du faire non-humain et s'entremêler à la phusis ${ }^{18}$ que l'intérêt se confirme d'employer la notion sur le terrain des habitats autonomes. La poiesis est en effet utilisée en grec ancien dès l'époque pré-classique pour désigner un certain nombre de productions et processus naturels tels que la croissance des céréales, la formation des étoiles ou encore la fabrication du miel par les abeilles (Liddel et al. 1996 : 1427-1429). Par ailleurs, au dix-neuvième siècle, on voit la notion resurgir dans le vocabulaire biologique et médical pour désigner un ensemble de processus et de fonctionnements métaboliques, sens qui voyagera à travers les sciences humaines au vingtième siècle jusqu'à désigner chez Heidegger et plus encore chez Maturana et Varela une dynamique d'auto-organisation propre aux êtres vivants (Heidegger, 1958: 16-17; Maturana, Varela, 1980 : xviii). Ce sens se diffusera ensuite dans les sciences sociales par le biais notamment de Félix Guattari qui, s'appuyant notamment sur les travaux de Maturana et Varela, affirme dans Les Trois écologies une conception de la poiesis élargie au-delà de l'humain en définissant l'autopoïesis comme "capacité reproductive " traversant les «machines sociales, écologiques et [...] [esthétiques]» $(1989: 49,60)$. Enfin, depuis la fin des années 1990, certain.e.s auteur.e.s des courants néomatérialistes et écocritiques mobilisent épisodiquement la poïesis "comme 'faire' organique soumis aux contraintes de l'évolution et à celles de la techne mais dépassant aussi largement cette dernière par son ouverture au hasard et son articulation à l'inventivité sémiotique du vivant» (Breteau, 2019:142). La conjonction et la cohérence de ces trajets légitiment alors le réinvestissement de la poïesis pour désigner le type de faire " organique ", ouvert au hasard et au vivant et donc à la fois humain et non-humain, que nous voyons se déployer à travers les habitats autonomes et qui en constitue la spécificité (Breteau, 2018 : 55-68).

Dans un écho à Michel de Certeau qui évoquait la poïssis comme un «type de production de l'ordre de l'émergence singulière, spontanée et indisciplinée » (Certeau, 1980 : xxxvii), l'un des premiers traits marquants de ce type de faire se trouve dans sa manière de se répandre, par proliférations et extensions successives. Ceci apparaît nettement chez Olivier Jullian, par exemple à travers une image située à l'articulation de deux façades du bâtiment (figure 9). 
Figure 9 : Une prolifération organique

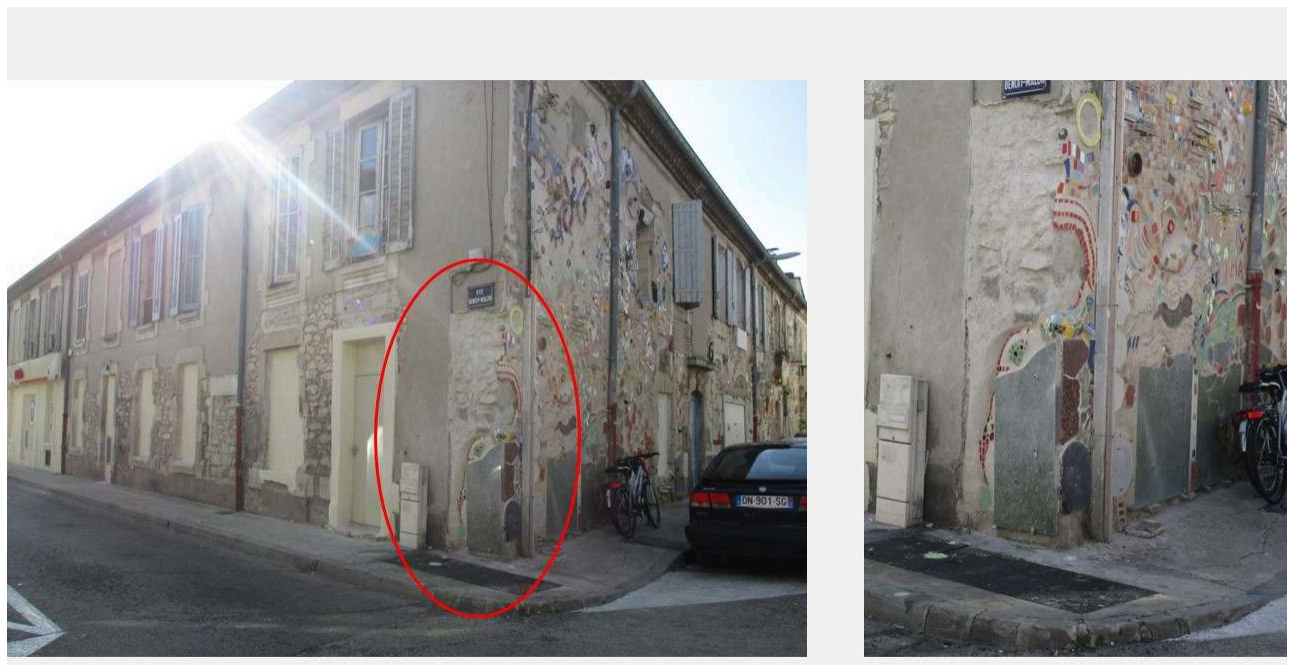

Source/crédit : Breteau, 2015

L'oiseau posé sur la façade de droite venant tendre le cou pour picorer sur la façade de gauche montre en effet la façon dont les fresques viennent, telles des entités naturelles et organiques qui la contamineraient, "grignoter " la maison. Ceci se voit illustré par un autre motif minuscule que l'on retrouve sur la façade « picorée » par l'oiseau.

Figure 10 : Des signes de contamination

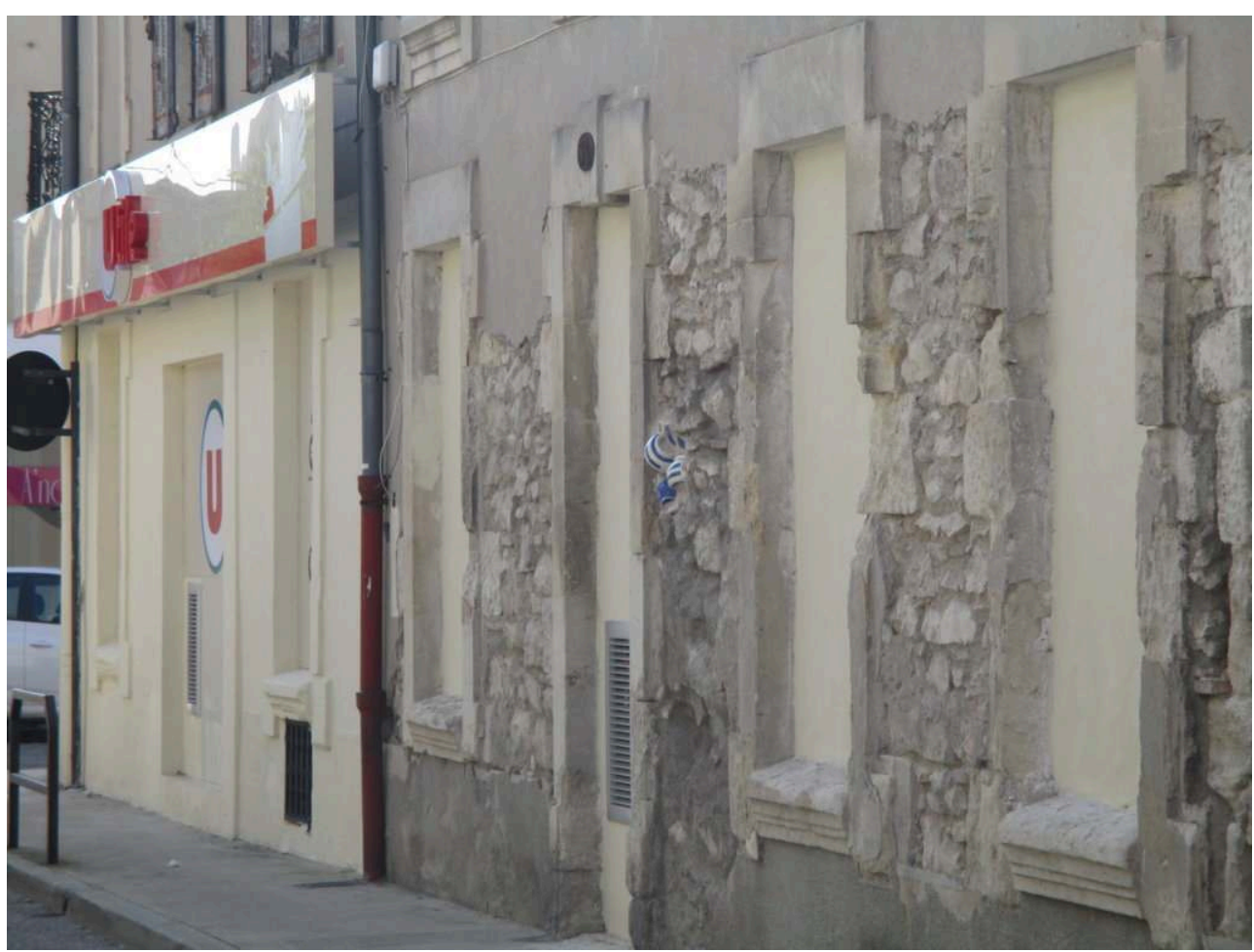

Source/crédit : Breteau, 2015 
Positionné au beau milieu d'un mur relativement nu et encore indemne de fresques, ce motif apparaît comme un "germe" ou comme une herbe folle, une petite plante adventice ayant poussé là par hasard (figure 10). Ainsi, les interventions d'Olivier Jullian ne suivent pas de logique linéaire mais procèdent plutôt par implantations arbitraires, colonisant le bâtiment par petits enclos ou " noyautages » successifs. Loin des théories hylémorphiques selon lesquelles la vie contenue seulement «en puissance " dans la matière doit être "actualisée » par des formes extérieures à elle (Aristote, 1994), ce type de faire se manifeste donc sous la forme de processus de croissance et d'excroissance organiques, semblant répondre aux manifestations d'une matière animée et habitée par une vie propre. Dans la cage d'escalier qui est l'un des rares espaces intérieurs "contaminés» par les fresques, une branche fait ainsi irruption à travers le mur, semblant mue là encore par une « poussée » interne (figure 11).

Figure 11 : Un faire qui « pousse » au travers

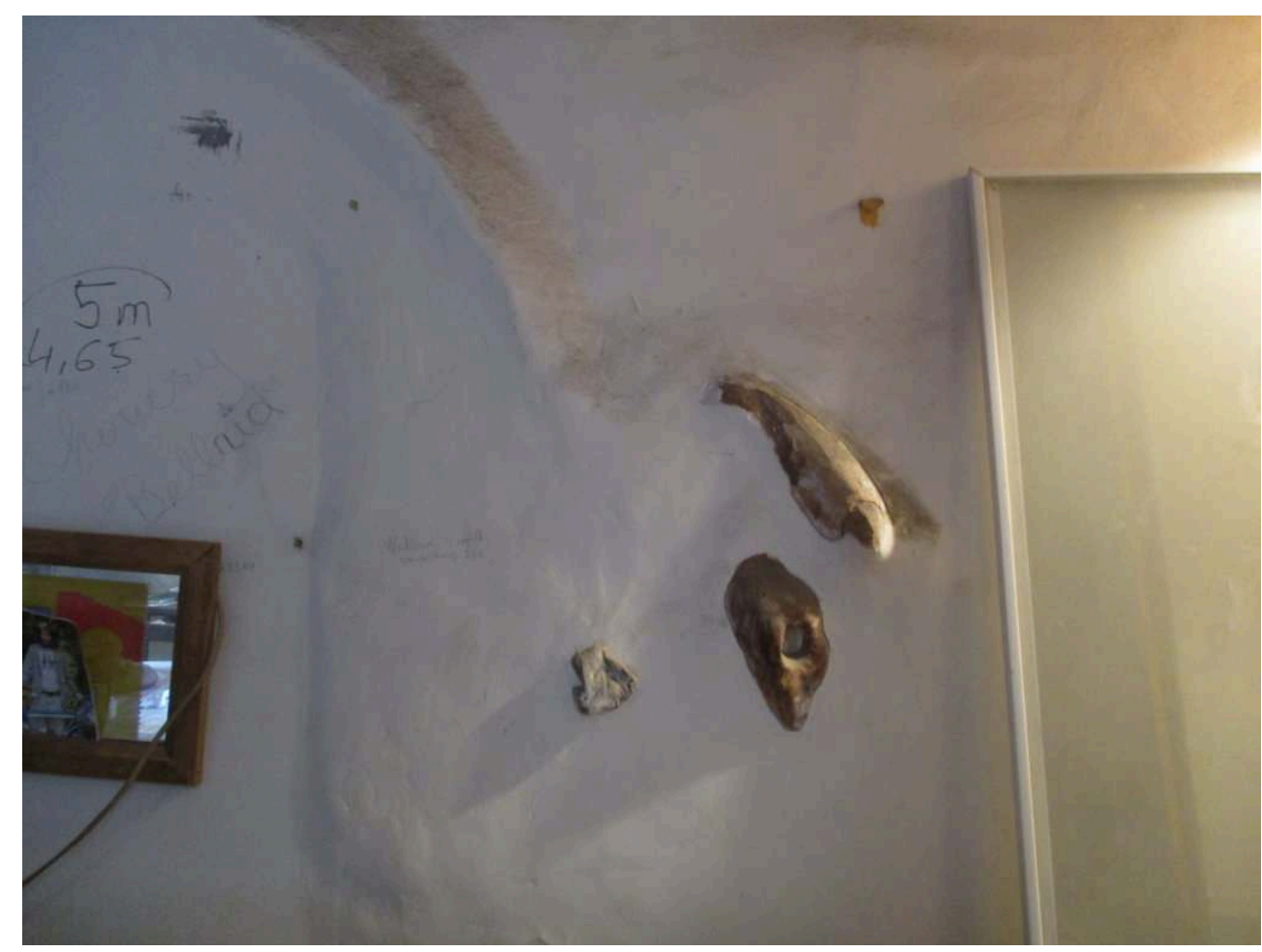

Source/crédit : Breteau, 2015

25 Ce type de faire organique se décline aussi à travers les formes représentées sur la façade par Olivier Jullian. On y retrouve ainsi de multiples sujets végétaux ou animaux, dont notamment la tortue, cet animal dont la carapace organique fait référence à la dimension syncrétique des façades de la maison ${ }^{19}$ (figure 12). 
Figure 12 : Sujets animaux et végétaux
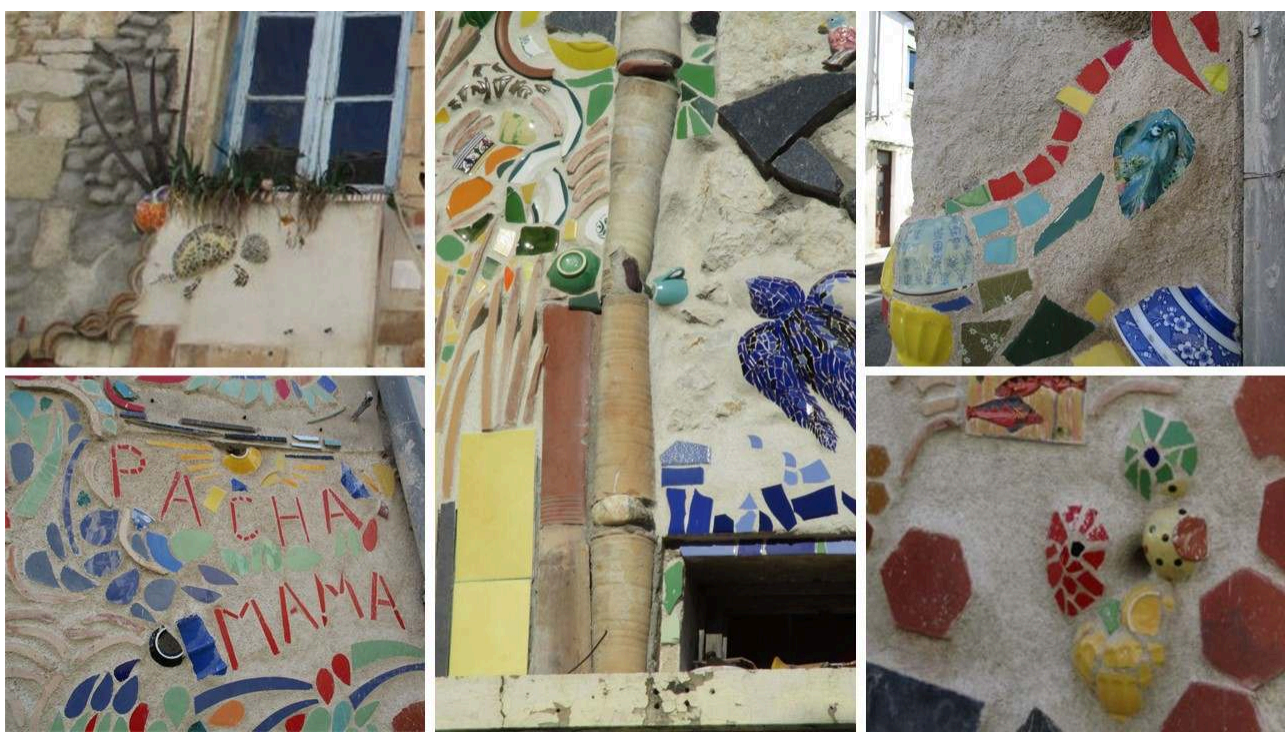

Source/crédit : Breteau, 2015

La figure du faucheur-semeur (figure 13) est également lourde de sens. Rappelant que le bâtiment était au xviie siècle un bâtiment agricole, elle se fait aussi le symbole d'un mode de production localisé certes dans le geste humain mais aussi et surtout, à travers la figure du semeur de graines, dans la puissance de vie ou poïesis propre de ce qu'il manipule. Cette dernière se voit notamment reflétée dans l'épaisseur multicolore et constellée de motifs de la tige "patchwork » sur le point d'être fauchée, qui n'est pas sans rappeler le motif textile de la toile de Pénélope. Le type de faire singulier de la poïesis prend dès lors la forme d'un "métier à tisser " aux prises avec la «toile du vivant $»^{20}$. Ainsi, les motifs naturels, échos d'un type de faire organique, se multiplient en lien direct avec les motifs textiles, eux-mêmes échos des liens physiques et métaboliques que les habitants autonomes recréent entre leur maison et leur environnement. 
Figure 13 : La figure du faucheur-semeur

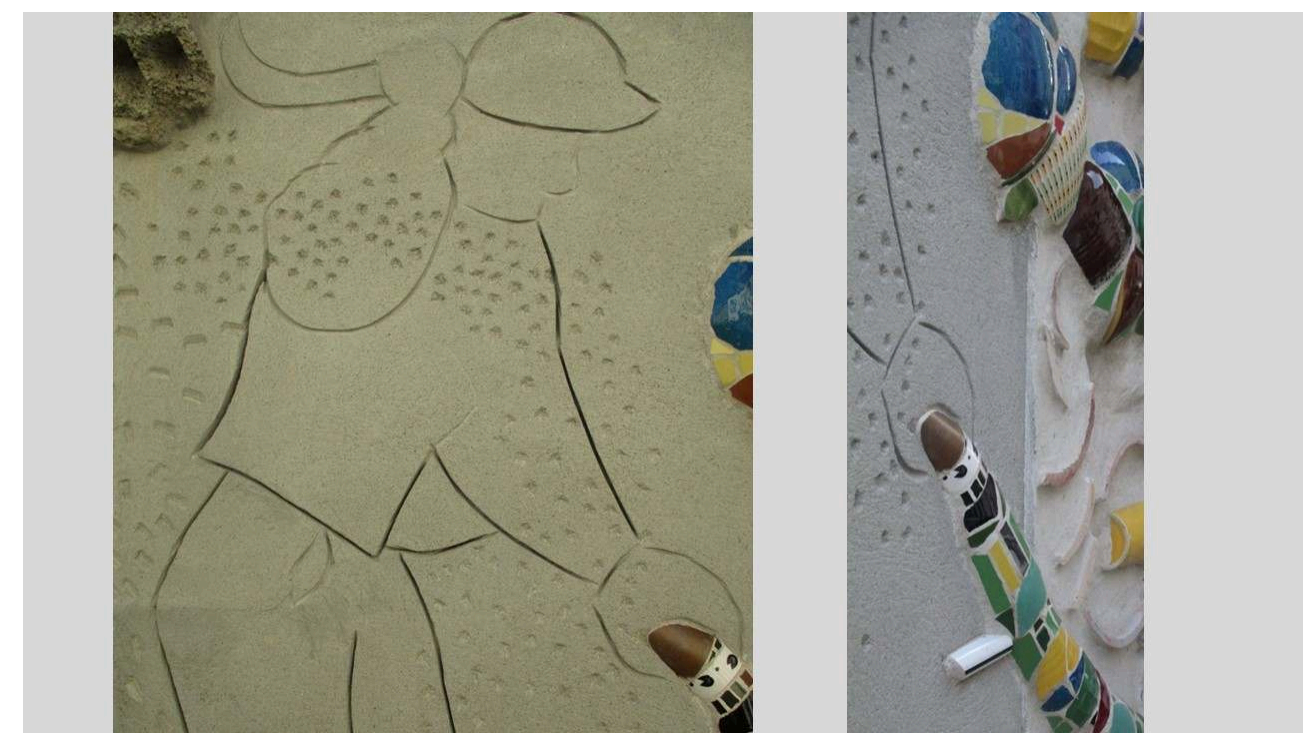

Source/crédit : Breteau, 2015

Toutefois, le faisceau d'éléments le plus significatif pour caractériser le type de faire organique déployé dans le lieu est sans doute à trouver dans la façon dont Olivier Jullian lui-même décrit sa manière de procéder. Ce dernier reconnaît en effet travailler sans plan précis, de manière intuitive et imprévisible. De la même façon que tout a commencé par la volonté de recycler, ses idées lui viennent « en tournant ${ }^{21}$. Non pas avec son rouet comme Pénélope, mais avec son balai : « tu vois, souvent, je ne sais pas quoi faire. Eh bien je vais balayer, nettoyer, ranger. Et puis au bout d'une heure que je tourne dehors, l'idée vient. Du coup j'ai une grande joie ${ }^{22}$. Par ailleurs, selon ses propres mots, sa première envie a été «de faire des arbres et des plantes ${ }^{23}$. Olivier Jullian arrose d'ailleurs régulièrement, quand il travaille, la façade du bâtiment - pour la rendre plus malléable et facile à travailler, d'une part, mais aussi comme s'il y avait là de vraies plantes, quelque chose d'organique qui demandait à être arrosééc ${ }^{24}$.

Ainsi, comme ces propos en témoignent, c'est un temps de latence biologique, organique, occupé à des tâches physiques et productives qui apparaît aussi comme le temps gestateur d'images et de métaphores. La poïesis productive et prosaïque s'avère, chez Olivier Jullian, entremêlée et nécessaire à la poïesis poétique et langagière ${ }^{25}$. Celuici déplore d'ailleurs la disparition ou le rétrécissement dans la société actuelle de ces temps de latence : « ça rejoint beaucoup la réalité sociale dans laquelle je vis, toutes les critiques qu'on peut faire du système. Il y a plus de temps pour balayer, plus le temps pour réparer ${ }^{26}$. Bien entendu, ce dernier verbe de " réparer " peut être entendu aussi bien dans un sens littéral que figuré. À travers lui, les propos d'Olivier Jullian rentrent en résonance avec cette toile de Pénélope qui, au-delà de prendre en écharpe le bâtiment, semble également circuler dans l'ensemble de son discours - la « réparation » étant en effet l'œuvre primordiale et continuelle du rouet de Pénélope, qui retisse le jour les portions de toile qu'elle a détruites la nuit.

Enfin, un dernier trait essentiel de la poïsis d'Olivier Jullian est lié à son caractère foncièrement hybride - celle-ci n'étant ni tout à fait de l'artisanat, ni tout à fait du bricolage, ni tout à fait de l'art, et en même temps un peu tout à la fois. En réalité, le faire qui se déploie sur les façades du bâtiment est à la croisée de différentes 
agentivités ${ }^{27}$ : celle d'Olivier Jullian bien sûr, mais aussi celle des tas de matériaux et matières qui suggèrent et font signes vers de nouveaux agencements et associations (Lévi-Strauss, 1962:30-31; Merleau-Ponty, 1969: 90-91). Rentrent également en compte l'agentivité de la maison elle-même et de son pouvoir "adhésif» et « enchanteur» (Gell, 2009 : 92, 103) ainsi que celle des passants et habitants du quartier qui viennent alimenter le pouvoir de la «maison-aimant " par leurs gestes d'offrande et leurs apports spontanés. Ce faisant, ils donnent corps à ce que l'on peut considérer comme une "poïesis urbaine ", "vague " matérielle à la croisée des individualités qui, venant former ses propres tas aux abords de la maison, se rapproche un peu de la « laisse » que la mer apporte sur les plages ${ }^{28}$.

\section{Des enchevêtrements ontologiques}

Toutefois, cette poiesis que nous venons de décrire engendre aussi un autre type de tissages. Venant compléter et prolonger les entrelacements métaboliques et esthétiques que nous avons évoqués dans les deux premières parties de cet article, ces tissages sont de nature ontologique et se déclinent à travers trois principaux aspects. Ils se manifestent tout d'abord par un phénomène de mélanges entre l'intérieur et l'extérieur. Ainsi cette branche d'arbre intégrée sans transformation à l'escalier de la pièce principale pour faire office de rampe, ou inversement ces fragments de vaisselle cassée encastrés dans la façade extérieure. Le meilleur exemple est à trouver sans doute dans cette inscription, "habiter le temps » (figure 14), dont Olivier Jullian a fait en sorte qu'elle puisse être vue aussi bien depuis l'intérieur que depuis l'extérieur du bâtiment - comme si le fait même d'« habiter » consistait à vivre cette réversibilité, à voir l'intérieur devenir extérieur et l'extérieur devenir intérieur.

Figure 14 : Habiter le temps, figure du mélange entre intérieur et extérieur

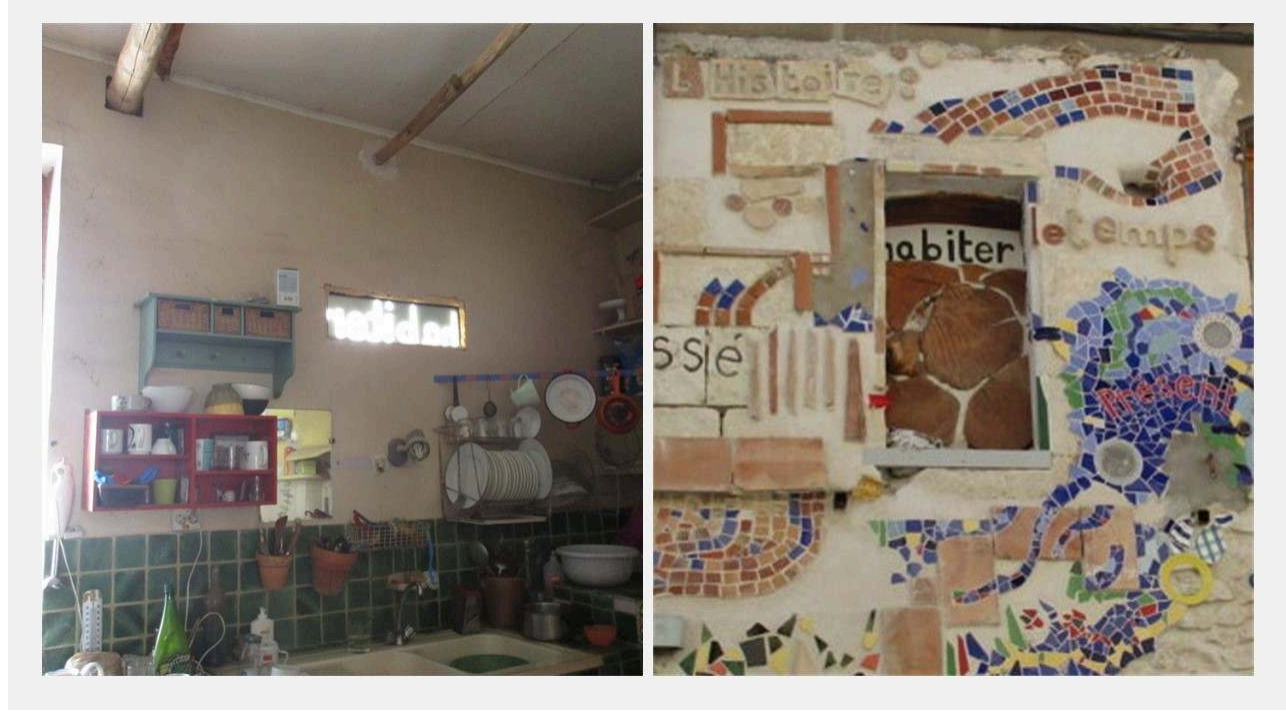

Source/crédit : Breteau, 2015

Cependant, les enchevêtrements ontologiques opérés par la poïesis autonome se traduisent aussi par des mélanges entre les mondes humain et non-humain. Ceux-ci se manifestent par des figures hybrides telles que cet homme-oiseau affublé d'un nez-bec 
représenté sur la façade, mais aussi celle de ce tronc-cadran, constitué de deux formes imbriquées du passage du temps : l'une, non-humaine, matérialisée par cette section de tronc incrustée dans la façade, avec ses multiples cercles concentriques marquant l'écoulement des années à même le corps de l'arbre; et l'autre, humaine, à travers cette aiguille blanche peinte par Olivier Jullian sur le tronc qui transforme tout à coup celuici en cadran d'horloge, instrument de mesure d'un temps cette fois bien humain (figure 15).

Figure 15 : L'homme-oiseau (à gauche) et le tronc-cadran avec son aiguille blanche (à droite)
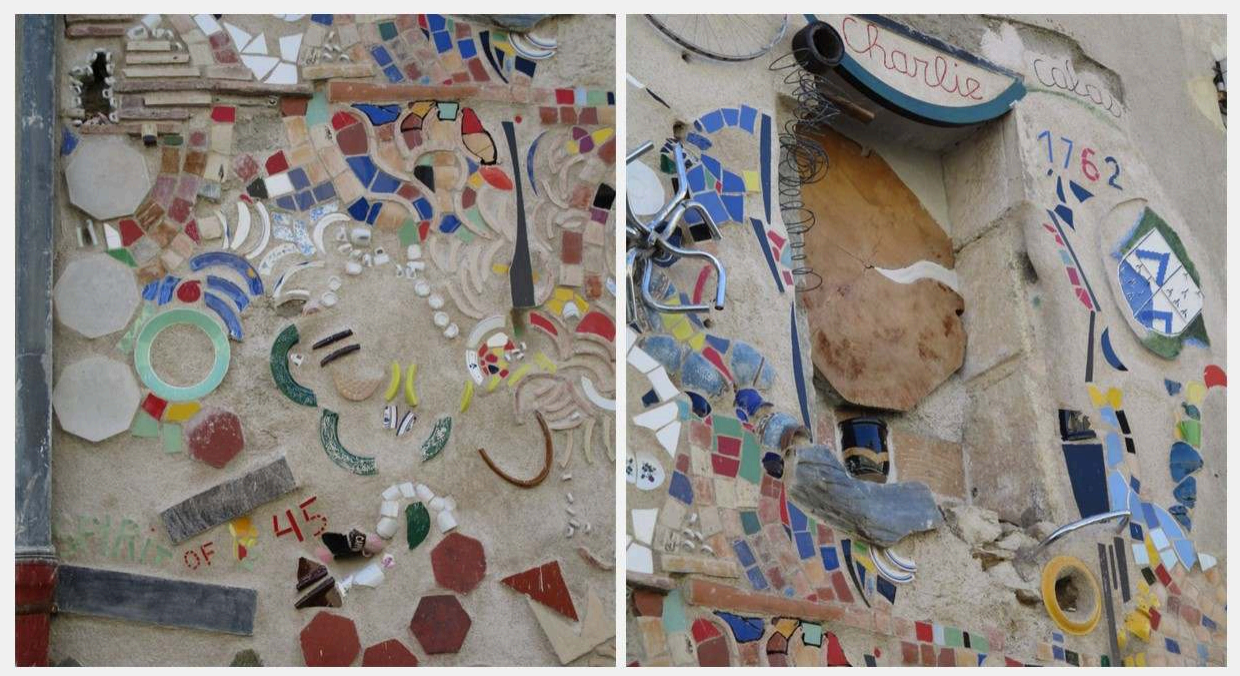

Source/crédit : Breteau, 2015

Enfin, ces bouleversements ontologiques se traduisent aussi par des tissages entre matière et signification. Ils marquent une resémantisation de la matière via des métaphores ancrées dans le corps physique de la maison. Celui-ci se voit notamment doté d'une expressivité propre, non seulement à travers les inscriptions verbales que l'on trouve sur les murs mais aussi sous la forme de ces petites mains qui apparaissent sur la façade et semblent prêter à la maison tout entière une agentivité et le pouvoir de « faire signe » (figure 16). 
Figure 16 : Une maison qui « fait signe »

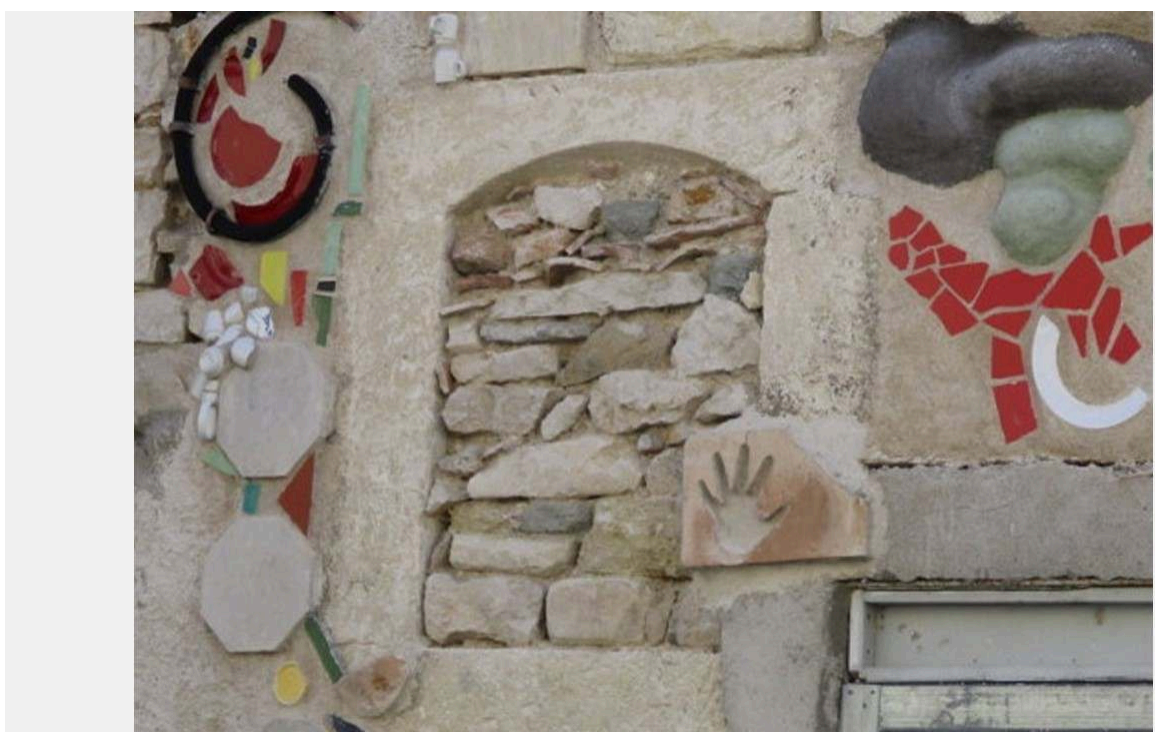

Source/crédit : Breteau, 2015

\section{Conclusion}

L'étude de cas que nous venons de mener nous permet de tirer plusieurs enseignements. Nous avons vu tout d'abord que les activités de recyclage urbain auxquelles se voue Olivier Jullian s'insèrent dans la tentative de retisser les fils d'une toile du vivant dénouée par les ravages écologiques de la société thermo-industrielle ainsi que dans l'ambition de réinscrire l'habitat dans cette toile.

Cependant, un autre enseignement apparait lorsque l'on compare cette "maisonstock» urbaine avec le reste des habitats autonomes en grande partie ruraux qui composent notre échantillon ${ }^{29}$. Dans le cas de ces derniers, le milieu naturel qui les environne et les échanges métaboliques nombreux qui se remettent en place font que les signes y prolifèrent également mais ne s'agrègent pas, ne s'entassent pas comme dans la maison d'olivier Jullian. À l'inverse, ils s'échelonnent dans l'espace, s'intègrent et se répartissent selon une organisation réticulaire, en des " points-clefs » (Simondon, 1958 : 165). Dans le cas que nous avons étudié, les agentivités qui se manifestent celles d'Olivier Jullian, des stocks, de la maison, de la ville - apparaissent comme "sauvages ", dans le sens où elles sortent des flux et infrastructures de recyclage et traitement prévues par la mégamachine industrielle, mais aussi parce qu'elles s'affirment comme spontanées, imprévisibles, élisant d'elles-mêmes leur habitat.

Cependant, parce que la toile des échanges support de l'autonomie se retrouve chez M. Jullian contrainte spatialement par la ville et par le manque de nourriture, elle ne peut se déployer complètement et se replie du même coup sur elle-même, se gonflant en une sorte d'excroissance et de "cancer» de poésie matérielle. Dès lors, si la "technologie de l'enchantement" que l'on observe dans la maison d'olivier Jullian donne bien à entendre un "chant", celui-ci s'avère relativement ambigu et ressemble sans doute davantage, lancé en milieu urbain dans la quête d'une autonomie impossible, à un « chant du cygne »... 


\section{BIBLIOGRAPHIE}

ARISTOTE (1994), De l'âme, texte établi par Antonio Jannone, traduction et notes de Edmond Barbotin, Paris, Gallimard.

BAILLY E. (2016), « Les paysages urbains en mal d'émotions », Carnets de géographes, no 9, http:// journals.openedition.org/cdg/594, consulté le 13 juillet 2019.

BERQUE A., DE BIASE A., BONNIN P. (dir.) (2012), Donner lieu au monde : la poétique de l'habiter. Actes du colloque de Cerisy-la-Salle, Paris, Donner Lieu.

BLANC N. (1995), La Nature dans la cité, Thèse de doctorat en géographie, Université PanthéonSorbonne Paris I.

BLANC N. (2010), « L'Habitabilité urbaine », in Coutard O., Lévy J. P. (dir.), Écologies urbaines, Paris, Economica Anthropos, pp. 169-83.

BLANC N., LAMARCHE T. (2015), « Services écosystémiques culturels : habitabilité, forme, gouvernance ", présentation réalisée dans le cadre du programme Éphèse, accompagnement par le LADYSS du Ministère de l'Écologie et du Développement Durable sur les services écosystémiques culturels.

BLANC N., BRETEAU C., GUEST B. (2017), « Pas de côté dans l'écocritique francophone », in FinchRace D., Weber J. (dir.), « French Ecocriticism / L'Écocritique française », L'Esprit Créateur, vol. 1, no. 57 , pp. $123-138$

BOUVET R. (2015), Vers une approche géopoétique : Lectures de Kenneth White, Victor Segalen, J.-M. G. Le Clézio, Québec, Presses de l'Université du Québec.

BRETEAU C. (2018), POEME : la Poïesis à l'Ère de la Métamorphose, Thèse de doctorat en géographie culturelle et esthétique environnementale, Université de Leeds (Royaume-Uni).

BRETEAU C. (2019), « POÈME : la Poïesis à l'Ère de la Métamorphose », Ecozon@, vol. 10, no. 1, pp. 136-163.

CAPRA F. (2003), La Toile de la vie: une nouvelle interprétation scientifique des systèmes vivants, Monaco, Éditions du Rocher.

CERTEAU (de) M. (1980), L'Invention du quotidien, t. 1, Paris, Gallimard.

COLLOT M. (2014), Pour une géographie littéraire, Paris, José Corti.

DARDEL E. (1952), L'Homme et la Terre. Nature de la réalité géographique, Paris, Presses Universitaires de France.

Dictionnaire Le Petit Robert (1986), sous la direction de Josette Rey-Debove et Alain Rey, Paris, Le Robert.

DICKS H. (2011), « The Self-Poeticizing Earth : Heidegger, Santiago Theory and Gaia Theory », Environmental Philosophy, vol. 8, no 1, pp. 41-61.

DOLPHIJN R., VAN DER TUIN I. (dir.) (2012), New Materialism. Interviews \& Cartographies, Ann Arbor, Open Humanities Press.

FRIZOT M. (dir.) (2012), Chaïm Soutine, l'ordre et le chaos, Paris, Hazan.

GELL A. (2009), L'Art et ses agents, une théorie anthropologique, Dijon, Les Presses du Réel.

GUATTARI F. (1989), Les Trois Écologies, Paris, Galilée. 
GUEST B. (2003), Écritures révolutionnaires de la nature au XIXe siècle. Géographie et liberté dans les essais sur le cosmos d'Alexander von Humboldt, Henry David Thoreau et Élisée Reclus, Thèse de doctorat en littérature, Université Michel de Montaigne Bordeaux 3.

HEIDEGGER M. (1958), Essais et Conférences, Paris, Gallimard.

INGOLD T., HALLAM E. (dir.) (2007), Creativity and Cultural Improvisation, Oxford/New York, Berg.

JAUSS H. R., SHAW M. (1982), « Poïesis », Critical Inquiry, vol. 8, no 3, pp. 591-608.

LEFEBVRE H. (1970), La Révolution urbaine, Paris, Gallimard.

LE GALL J., ROUGE L. (2014), « Oser les entre-deux », Carnets de géographes, no. 7, http:// journals.openedition.org/cdg/496, consulté le 13 juillet 2019.

LEVI-STRAUSS C. (1962), La Pensée sauvage, Paris, Plon.

LIDDEL H., SCOTT R., JONES H. S. (1996), A Greek-English Lexicon, Oxford, Oxford University Press.

LOUBES J.P. (2010), Traité d'architecture sauvage, Paris, Éditions du Sextant.

MATURANA H., VARELA F. (1980), Autopoiesis and Cognition : The Realization of the Living, Dordrecht/Boston, D. Reidel.

MEILLON B., LAUWERS M., PERRIN C., DURAND-ROUS C. (2018), « Réenchanter le sauvage urbain ", Appel à contributions du colloque international à Perpignan au Palais des Rois de Majorque organisé du 11 au 14 juin 2019 par l'Atelier de Recherche en Écocritique et Écopoétique, sous l'égide du CRESEM, Université de Perpignan Via Domitia (UPVD).

MERLEAU-PONTY M. (1969), La Prose du Monde, Paris, Gallimard.

MICHEL L. (2010), «Crise de la poésie ? Le poétariat selon Jean-Claude Pinson », Les Temps Modernes, vol. 657, no. 1, pp. 247-59.

MOREL-BROCHET A., ORTAR, N. (2012), La Fabrique des modes d'habiter. Homme, lieux et milieux de vie, Paris, L'Harmattan.

PLATON (2007), Le Banquet, Paris, Flammarion.

PRUVOST G. (2013), « L’Alternative écologique. Vivre et travailler autrement », Terrain, no 60, pp. 36-55.

SAUSSURE (de) F. (2005), Cours de linguistique générale, Paris, Payot.

SIMONDON G. (1958), Du mode d'existence des objets techniques, Paris, Aubier.

THOREAU H. D. (1960), Walden (and Civil Disobedience), New York, The New American Library of World Literature.

WHITE K. (2009), Les Affinités extrêmes, Paris, Albin Michel.

\section{NOTES}

1. Nos italiques.

2. Voir par exemple le colloque international «Réenchanter le sauvage urbain » organisé à Perpignan au Palais des Rois de Majorque du 11 au 14 juin 2019 par l'Atelier de Recherche en Écocritique et Écopoétique, sous l'égide du CRESEM, Université de Perpignan Via Domitia (UPVD). Détails disponibles via le lien: https ://ecopoeticsperpignan.com/programme-reenchanter-lesauvage-urbain-reenchanting-urban-wildness/. 
3. Conformément à sa volonté de travailler à un "nouvel ordre métaphysique ", le nouveau matérialisme mobilise la notion d' "enchevêtrement " pour réfléchir à la croisée des concepts dualistes et faire apparaître entre eux, à l'appui de terrains ou de situations concrètes, de nouveaux agencements et tissus conceptuels. Selon ce principe, le terme d' "enchevêtrement " sera utilisé ici pour désigner une « disposition de choses [...] [engagées les unes dans les autres] de façon désordonnée » (Dictionnaire Le Petit Robert, 1986). Mais il sera aussi mobilisé, aux côtés des notions d' "entrelacement", de "rebouclage» ou de "tissage», pour se référer aux agencements nouveaux qui s'élaborent sur nos terrains entre intérieur et extérieur, matière et signification, humain et non-humain.

4. Thèse de géographie et études culturelles effectuée d'octobre 2015 à novembre 2018 sous la codirection de Nathalie Blanc (géographe, Ladyss, Paris), Nigel Saint et Claire Lozier (enseignantschercheurs en études culturelles françaises, Université de Leeds, R-U).

5. Les travaux dont nous rendons compte ici s'orientent certes vers une alliance de la poésie et de la géographie telle que les géopoétiques de Deguy, White ou Bouvet ont pu le proposer. Cependant, la géopoétique focalise largement son attention - non pas sur le domaine des cultures matérielles au niveau local de l'habitat quotidien - mais sur la poésie verbale et l'art envisagés comme la confrontation large de subjectivités " poètes » ou " artistes " aux grandes entités du monde. Afin de renforcer son potentiel éthique et politique, nous avons choisi pour notre part d'ancrer notre étude de la poïesis dans les cultures matérielles plutôt que dans des expériences artistiques détachées de la vie quotidienne, et préférons donc pour cette raison ne pas nous placer directement pour l'instant dans le prolongement des approches géopoétiques.

6. Recherches entreprises dans le cadre de la thèse de doctorat POÈME : la POïesis à l'Ère de la MEtamorphose (Breteau, 2018).

7. La notion de métabolisme est ici entendue comme l'ensemble des échanges d'énergie et de matière (destructeurs et créateurs, cataboliques et anaboliques) qui permettent à un organisme de se maintenir en homéostasie, soit en rapport équilibré avec son environnement.

8. Sur 24 habitats étudiés, 18 sont localisés en milieu rural hors village, 2 dans des villages ruraux, 1 en zone périurbaine, 2 en périphérie de village et 1 seul en milieu urbain. Dans cette nomenclature, la catégorie de "rural » exclut les habitats dans les villes, centre-bourgs de villages et zones périurbaines. En cas de doute, nous y incluons les terrains de statuts fonciers agricole, naturel ou « de loisir » (85\% de l'échantillon) et en excluons les terrains constructibles.

9. Voir note 6.

10. Un bâtiment bioclimatique est un bâtiment dont l'implantation et la conception prennent en compte le climat et l'environnement immédiat afin de réduire au maximum sa consommation énergétique.

11. Extrait de l'entretien réalisé avec Olivier Jullian le 13 novembre 2015.

12. Ibid.

13. Olivier Jullian incruste d'ailleurs également à la façade des " parefeuilles », ces carrelages en terre cuite qui étaient autrefois posés en traverse sous les tuiles d'un toit.

14. «L'aspect du [bâtiment] ne vaut plus seulement pour indiquer, au-delà de lui-même, des ustensiles à manier. L'action (...) [se refuse] aux abstractions de l'utile et n'entend pas sacrifier les moyens à la fin, l'apparence à la réalité. Tout compte désormais, l'usage des objets moins que leur aptitude à composer ensemble, jusque dans leur texture intime, un emblème valable du monde auquel nous sommes confrontés » (Merleau-Ponty, 1969 : 90-91).

15. Extrait de l'entretien réalisé avec Olivier Jullian le 13 novembre 2015.

16. Ibid.

17. Base de données «Indo-European Lexicon. PIE Etymon and IE Reflexes", https:// lrc.la.utexas.edu/lex/master/1098, consultée le 14 juillet 2019.

18. Nous entendons ici avec Heidegger la phusis comme "poïesis au sens le plus élevé », soit comme mode de production « [par lequel] la chose s'ouvre d'elle-même » (Heidegger, 1958 :16-7). 
19. Voir notamment à ce sujet Henry David Thoreau qui, dans Walden, évoque l'« architectural beauty " de maisons " that [have] gradually grown from within outward, out of the necessities and [...] life of the inhabitants ", comme une carapace ayant poussé sur le dos d'une tortue (1960:36).

20. Le motif de la «toile » est évoqué de manière constante à travers la littérature scientifique ou de vulgarisation pour évoquer le monde vivant (Capra, 2003).

21. Extrait de l'entretien réalisé avec Olivier Jullian le 13 novembre 2015.

22. Ibid. Notre accentuation.

23. Ibid.

24. Cette pratique n'est pas sans rappeler également l'habitude qu'avait le peintre Soutine d'arroser de sang frais les carcasses d'animaux écorchés qu'il peignait. Voir notamment Chaïm Soutine, Carcasse de bœuf, huile sur toile, 156,21 x 122,55 cm, Buffalo ; New York, Albright-Knox Art Gallery, vers 1925 (Frizot, 2012 :124-7).

25. Nous prenons ici la notion de « langage » dans son sens saussurien comme système de signes distinct du système strictement verbal de la « langue » (Saussure, $2005: 25$ ).

26. Extrait de l'entretien réalisé avec Olivier Jullian le 13 novembre 2015.

27. La notion d' "agentivité » est ici comprise comme capacité d'agir et de se manifester, soit comme une somme d'intentions, de pouvoirs et de contrôles partagée aussi bien par les êtres humains que par les forces du monde qui les entoure.

28. La laisse de mer désigne à la fois l'espace découvert sur le littoral entre les marées hautes et les marées basses, mais aussi les divers détritus, objets, algues, cadavres, coquillages, épaves qu'y déposent la houle et les courants de marée.

29. Voir note 7 .

\section{RÉSUMÉS}

En écho aux dynamiques actuelles qui voient converger géographes et littéraires autour d'objets " écopoétiques », nous proposons dans cet article d'examiner les enchevêtrements entre matière et signification qui se déploient dans un lieu urbain désormais célèbre, celui de la maison d'Olivier Jullian à Nîmes. Partie d'un énorme tas de tuiles dégagé lors de la réfection du toit, cette «maison-stock» occupant tout un îlot urbain a acquis au fil des ans une fertilité propre, à mesure que sont venus s'agréger sur ses façades et toitures des milliers de débris de vaisselle et fragments d'objets. Nourrie par la ville, et notamment par les habitants du quartier venant déposer aux abords de la maison tasses, assiettes cassées et rebuts divers, cette "maisonaimant " s'hybride avec son double rural, intégrant aussi de nombreux matériaux et objets que son propriétaire, ramène d'une seconde maison autoconstruite et autonome située en pleine campagne. Dans le cadre théorique des nouveaux matérialismes, cet article étudiera la façon dont se déploie à travers la maison d'Olivier Jullian un type de faire organique porteur d'une " autre » écopoétique, la « poïesis » se déployant non plus dans les textes mais dans le corps même de la ville, à partir d'un tissu de reconnexions métaboliques étroites. Nous verrons ainsi à travers le cas d'Olivier Jullian et de sa maison comment cette poïesis, marquée par des "poussées » imprévisibles et des proliférations spontanées, enchevêtre au sein de ses productions intérieur et extérieur, matière et significations mais aussi humains et non-humains, déterminant l'apparition d'une véritable « technologie de l'enchantement ». 
Echoing current dynamics that see geographers and literature researchers converge around "ecopoetical" objects, we propose in this article to examine the entanglements between matter and meaning that unfold in a now famous urban place, Olivier Jullian's house in Nîmes. Starting from a huge pile of roofing tiles that had to be "diminished" at all costs, this gigantic "housestock" occupying an entire urban island has acquired over the years a fertility of its own as thousands of crockery fragments have been added to its roofs and facades. Nourished by the city, and especially by the inhabitants of the district who are constantly bringing in cups, broken plates and various rubbish, the "house-magnet" also integrates many materials and objects that his owner brings back from his country house. Within the theoretical framework of the new materialisms, this article will study the way in which a specific type of "poiesis" unfolds throughout Olivier Jullian's house. Reevaluated as an organic type of making and as "another" ecopoetics, the latter unfolds not in texts but in the very body of the city from a tissue of narrow metabolic reconnections. Therefore, we will see with the case of Olivier Jullian and his house how poiesis, marked by unpredictable "surges" and spontaneous proliferations, intertwine in its productions a number of dimensions - interior and exterior, matter and meanings but also human and non-human - resulting in the deployment of a true "technology of enchantment".

\section{INDEX}

Thèmes : Carnets de recherches

Keywords : Self-sustaining Housing, Ecopoetics, Environmental Geography, Enchantment

Mots-clés : habitat autonome, écopoétique, géographie environnementale, enchantement

\section{AUTEUR}

\section{CLARA BRETEAU}

Chercheuse post-doctorante en géographie et esthétique environnementales

clara.breteau[at]gmail.com 